\title{
CLASSIFICATION OF APPENDICITIS BASED ON ULTRASOUND IMAGE
}

\author{
Kinsana Htwe ${ }^{1}$, Aung Soe Khaing ${ }^{2}$ \\ ${ }^{1}$ M.E Thesis Student, Department of Electronic Engineering, Mandalay Technological University, Mandalay, \\ Myanmar \\ ${ }^{2}$ Associate Professor, Department of Electronic Engineering, Mandalay Technological University, Mandalay, \\ Myanmar
}

\begin{abstract}
Ultrasound image can facilitate the physician to identify the cause of an enlarged abdominal organ. Ultrasound is no use the ionizing radiation way so it is useful for scanning pregnant patient. Ultrasound is available and inexpensive modality with the potential for highly accurate imaging in the patient suspected to have acute appendicitis. In order to detect the appendicitis from the ultrasound, an algorithm will be developed using MATLAB Image Processing Toolbox to identify the diagnosis of appendix solely based on ultrasound image. Ultrasound images of appendicitis are imported into MATLAB and are passed through a set of filters to remove background noise. Next, the filtered images are run through a set of segmentation algorithms and a set of edge detection algorithms which identifies and defines the region of interest. Various thresholding methods can be used for segmentation of the image. After image processing, measuring the appendicitis diameter image can be helpful for a better diagnosis.
\end{abstract}

Keywords: Ultrasound, Appendicitis, Image segmentation, Image detection, Euclidean distance

\section{INTRODUCTION}

Acute appendicitis is more serious than any other acute conditions for requiring urgent abdominal surgery in all age .Appendix is a narrow, worm-shaped blind end tube. It has about $8 \mathrm{~cm}$ in length and $0.5-1 \mathrm{~cm}$ width. The adult Appendicitis is a long, diverticulum averaging $10 \mathrm{~cm}$ in length that arises from the poster medial wall of the cecum in the region of $3 \mathrm{~cm}$ below the ileocecal valve. Appendicitis generally effect people aged between 10 and 30, but it can strike at any age [3]. The symptoms of appendicitis include pain in the lower right abdomen, nausea, loss of appetite, vomiting and fever. Infection and obstruction are the causes of appendicitis. Infection is like as a stomach infection that may have found its way to cause the appendicitis. The next cause of appendicitis, obstruction, a hard piece of stool may have got trapped in the appendix and the bacteria in the trapped stool may have infected the appendix. Ultrasound is used to identify the diagnosis of acute appendicitis [4]. The diagnosis of appendicitis can be found by the ways such as Ultrasound, CT scan (Computer Tomography), MRI (Magnetic Resonance Imaging), Urine test. Ultrasound is available and inexpensive modality with the potential for highly accurate imaging in patient suspected to have acute appendicitis. Computer Tomography (CT) is also used to find the diagnosis of acute appendicitis. The outer diameter of appendicitis is $6 \mathrm{~mm}$ or more. Visualization of the appendix in patients who denied history of appendectomy was considered as true-positive results and non-visualization of the appendix in patients who had undergone appendectomy was considered as true-negative results. Visualization of the appendix in patients who had undergone appendectomy was considered as false-positive results and non-visualization of the appendix in patients who denied history of appendectomy was considered as falsenegative result [1].

In order to improve the accuracy of detection acute appendicitis, the requirement of image processing methods are an important process to determine the better assessment of ultrasound image. Many image processing methods are used to enhance ultrasound image in order produce better and clear image. The occurrence of noise in an image is caused by the acoustic nature of surrounding tissue. So, filtering techniques are required to remove the noise from the image. Image segmentation method using histogram thresholding are used to segment out the image and canny edge detector are used for edge detection method, one of the image processing methods [8]. 


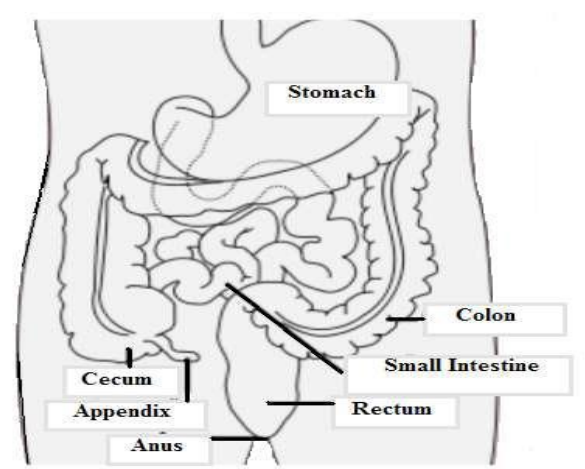

Fig-1: Position of appendix in human body

\section{METHDOLOGY}

This section will discuss the overall methodology of this study. The detection of the ultrasound appendix images has the following steps. These are: at first the image is captured from the ultrasound. If the image is a color image, it will convert the gray scale image. The second step is image preprocessing; the image of label, masks are removed from the image. Next step is image enhancement; noise from the preprocessed image is removed using median filter. The later step is image segmentation; the required portion is segmented out and detected the edge of the appendicitis. Finally, Euclidean distance formula is used to measure the diameter of appendix [6].

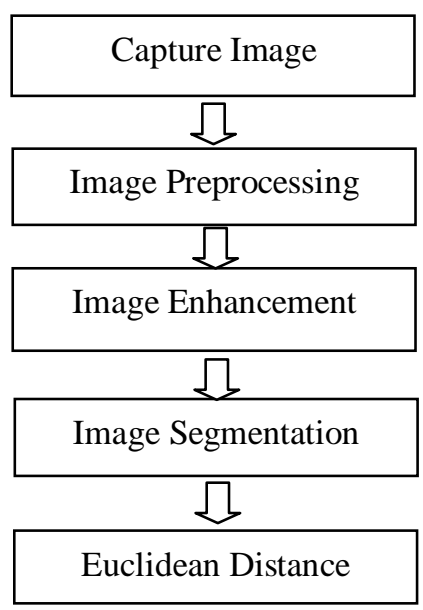

Fig-2: Process Flow Chart

\subsection{Capture Image}

Image Capturing is a process to acquire the digital image into MATLAB. In this module medical image was given as input and all types of medical images can be acquired in this module [7] .Images of a patient obtained are displayed as an array of pixels and stored in memory. Large matrix will specify a black and white image and 0 corresponds to black and 1 to white. A grey scale image can be specified by giving a large matrix whose entries are numbers between 0 and 1 . A black and white image is specified by giving a large matrix with integer entries. The lowest entry corresponds to black and the highest to white. Ultrasound image from the database is obtained through imread command in mat lab. The exact path of the image should be given as the argument to imread command. The image of the patient is displayed as an array of pixels and stored in the memory. In mat lab, captured image is displayed using imshow command by passing a variable as the argument and the image can also be displayed in the image viewer using imread command [5].

\subsection{Image Preprocessing}

The objective of the preprocessing is to improve the image data quality by suppressing undesired distortions and to enhance the required image features for the further processing. The preprocessing technique can be applied to eliminate the irrelevant data contains in the image. Moreover, these technique can be removed the incomplete, noisy and inconsistent data from the image.

\subsubsection{Median Filtering}

Median filtering is a nonlinear digital filtering technique. So, it is very widely used in digital image processing because of the under certain conditions, it preserves edges while removing noise, replaces each pixel by the median of the window area pixels, is more effective against impulse noise. Then, this filter can be used to remove the high frequency component in the image.

\subsubsection{Average Filtering}

Average filter can be replaced each pixel by the average of the window area pixels. It has the effect of smoothing image. The most simple low-pass filter is the local averaging operation. The main effect of low-pass filter is a blurring. The size of the kernel is $(2 \mathrm{~N}+1) \times(2 \mathrm{~N}+1)$ :

$$
h(k, l)=\left\{\begin{array}{cl}
\frac{1}{(2 \mathrm{~N}+1)^{2}} & -\mathrm{N} \leq \mathrm{k}, 1 \leq-\mathrm{N} \\
0 & \text { otherwise }
\end{array}\right.
$$

For $\mathrm{N}=1$, the convolution kernel is given by:

$$
\mathrm{H}=\frac{1}{9}\left[\begin{array}{lll}
1 & 1 & 1 \\
1 & 1 & 1 \\
1 & 1 & 1
\end{array}\right]
$$

\subsection{Image Enhancement}

The principal objective of enhancement techniques is to process an image so that the results are more suitable than the original image for a specific application. The result image of 
the above step is enhanced by using the histogram equalization and adaptive histogram equalization.

\subsubsection{Histogram Equalization}

It is one of the contrast enhancement techniques. Image quality can be improved by altering histogram. All gray level used has a tendency to enhance image contrast. The goal is to increase the global contrast of images, especially when the usable data of the image is represented by close contrast values.

\subsubsection{Adaptive Histogram Equalization}

Unlike histogram, it operates on small data regions (tiles) rather than the entire image. And also contrast enhancement can be limited in order to avoid amplifying the noise which might be presented in the image. So, Adaptive histogram equalisation technique works significantly better than regular histogram equalization for most images.

\subsection{Image Segmentation}

Image segmentation is a vital method for most medical image analysis tasks. Segmentation is an important process to extract information from complex medical image. There are many image segmentation methods. They are Watershed segmentation, Otsu's thresholding, Region growing, graph cuts method, Histogram thresholding, and edge detection. Image segmentation refers to the process of partitioning an image into a group of pixels using Histogram thresholding method and canny edge detection method.

\subsubsection{Otsu's Thresholding}

Otsu's thresholding method is based on region homogeneity that can be measured using variance. Otsu's method selects the threshold value by minimizing the within-class variance or maximizing between-class variance. In this method, it selects the threshold by minimizing the within-class variance $(\sigma 2)$ or maximizing between class variance $(\sigma 2)$, given by (1) which reduced to as (2) which the term defines in (3) where $n$ represents the number of grey levels and $\mathrm{N}$ is the total number of pixel in the image. The between-class variance is defined as;

$$
\begin{gathered}
\sigma^{2}(t)=w_{0}(t) w_{1}(t)\left[\mu_{1}(t)-\mu_{2}(t)\right]^{2} \\
\sigma^{2}(t)=w_{0}(t)\left[1-w_{0}(t)\left[\frac{\mu-\mu_{1}(t)}{1-w_{0}(t)}-\frac{\mu(t)}{w_{0}(t)}\right]\right] \\
w_{0}(t)=\sum_{N}^{n} w_{1}(t)=1-w_{0}(t), \mu(t)=\sum t \frac{n}{N}
\end{gathered}
$$

\subsubsection{Histogram Thresholding}

Histogram thresholding is one of the image segmentation methods. Histogram thresholding method is the proposed thresholding method. It defines the threshold level that is multiplying the maximum gray level of an image with the normalized threshold value. The normalized threshold value is within the range of 0 to 1 . Therefore, the normalized threshold value is set as 0.2 along the process running. This method searches the edge direction by implementing non-maximum suppression to sharpen the edge.

\subsubsection{Edge detection}

The purpose of edge detection is to identify areas of an image where a large change in intensity occurs. Edge detection method is used for segmentation and also identification of objects in a scene. There are many edge detection methods such as Roberts, Prewitt, Sobel, Laplacian of Gaussian (LOG), Canny edge detector and so on. Among of them, canny edge detector is applied for the process. Canny edge detector is mainly refered to the group of the pixels that have strong changes and contain the useful information of identifying. Canny edge detection method is a modification of Sobel method. This method searches the edge direction by implementing non-maximum suppression to sharpen the edge. Compared to the other methods, Canny method provides good edge detection because of its good performance of single response to edge [9].

\subsubsection{Canny Edge Detector}

Canny edge detector uses linear filtering with a Gaussian kernel to smooth the noise in the image. Then, the edge strength and direction are calculated for every pixel in the smoothed image. The pixel that survive the non-maximal suppression thinning process are labeled as candidate edge pixels.

\subsubsection{Sobel Edge detector}

The Sobel edge detector uses the masks to approximate digitally the first derivatives $G_{x}$ and $G_{y}$. In other words, the gradient at the center point in a neighborhood is computed as follows by the Sobel detector:

$$
\begin{aligned}
\mathrm{g}= & {\left[G_{x}^{2}+G_{y}^{2}\right]^{1 / 2} } \\
= & \left\{\left[\left(z_{7}+2 z_{8}+z_{9}\right)-\left(z_{1}+2 z_{2}+z_{3}\right)\right]^{2}+\right. \\
& {\left.\left[\left(z_{3}+2 z_{6}+z_{9}\right)-\left(z_{1}+2 z_{4}+z_{7}\right)\right]^{2}\right\} }
\end{aligned}
$$

\subsection{Euclidean Distance}

The Euclidean distance is the straight-line distance between two pixels. It is used to find out the diameter of acute appendicitis. The outer diameter of appendicitis is measured 
the distance between the outer borders of muscle coat. Euclidean distance is the distance between two points of Euclidean space. The distance between two points is defined as the square root of the sum of the squares of the differences between the corresponding coordinates of the points [2].By mathematical expression,

$$
d(p, q)=\sqrt{\left(p_{1}-q_{1}\right)^{2}+\left(p_{2}-q_{2}\right)^{2}}
$$

\section{RESULTS AND DISCUSSION}

Table-1 Matlab Flow Chart for the overall process implementation

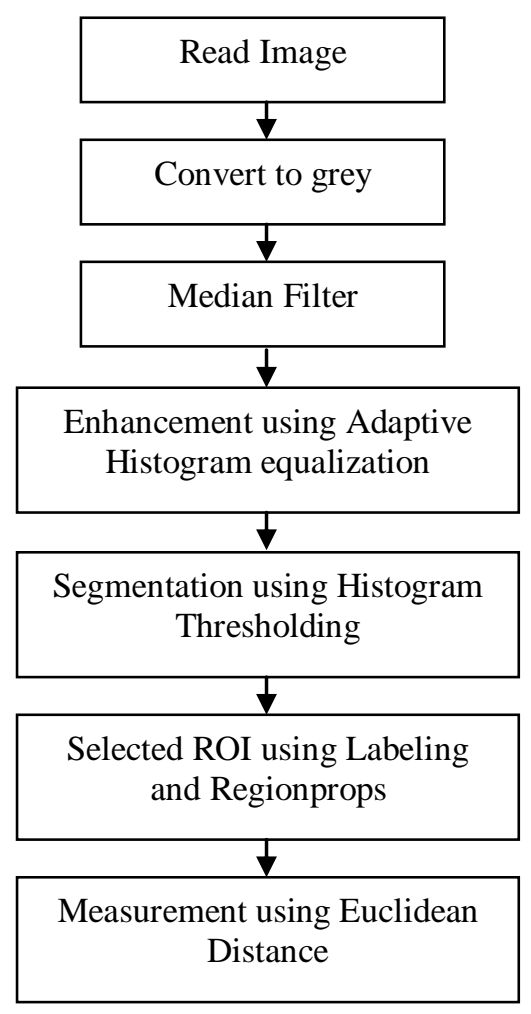

Table. 1 shows the overall implementation in this paper

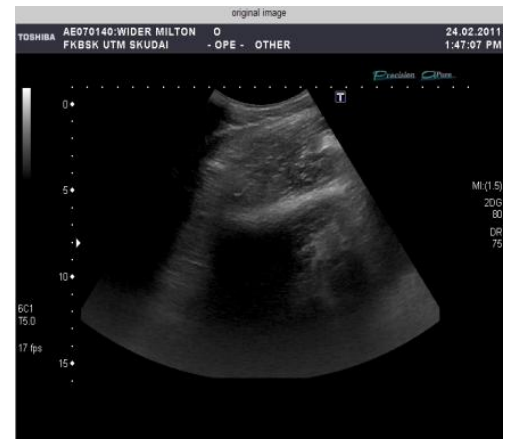

Fig-3: Original Ultrasound Image of Appendix

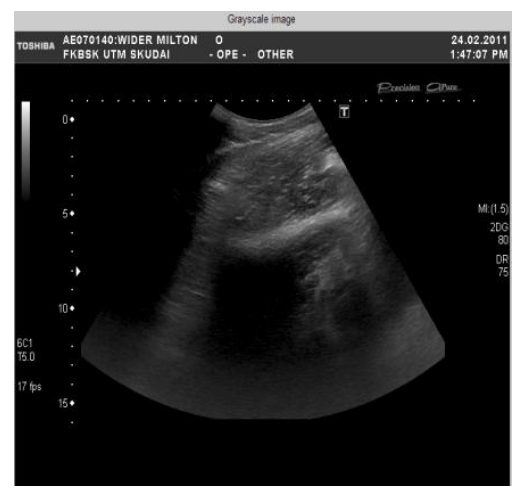

Fig-4: Gray Scale Image of Appendix Image

Fig (5) and Fig (6) are described the results of image preprocessing step. Median Filtering technique is appropiate for this research.

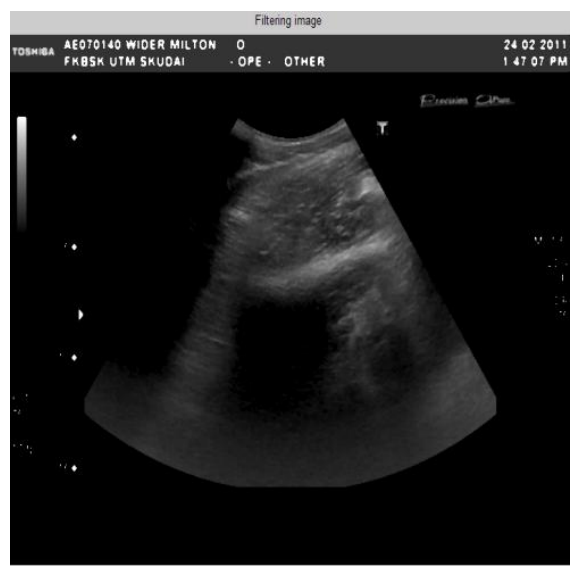

Fig-5: Result of median filtering image

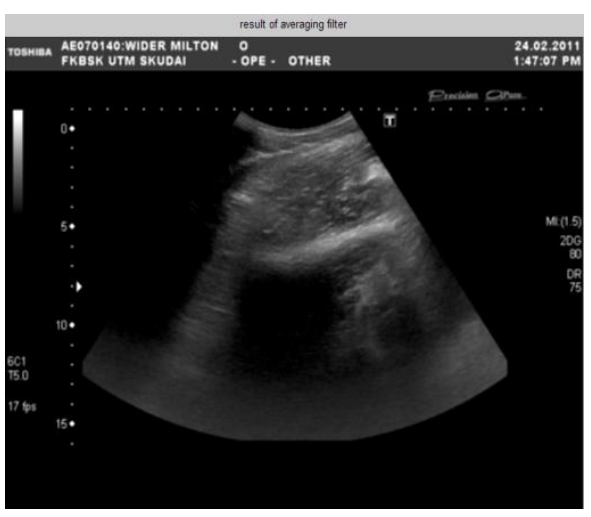

Fig-6: Result of average filtering image

Fig (7) and Fig (8) are shown as the outputs of the image enhancement step. Fig (7) describes the result using the histogram equalization method. Fig (8) is the result of using adaptive histogram equalization. 


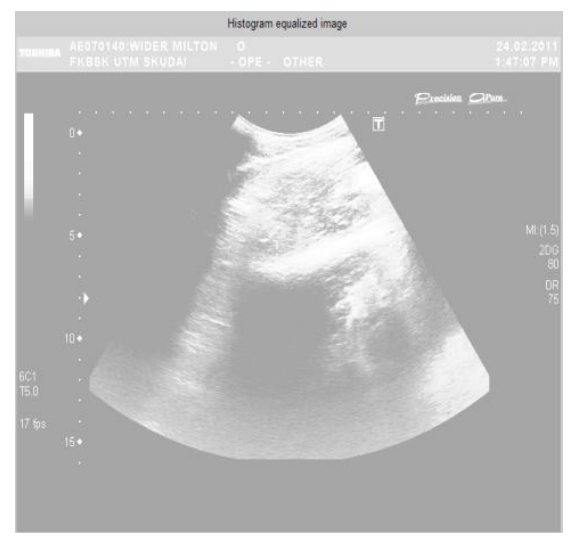

Fig-7: Result of Histogram Equalized Image

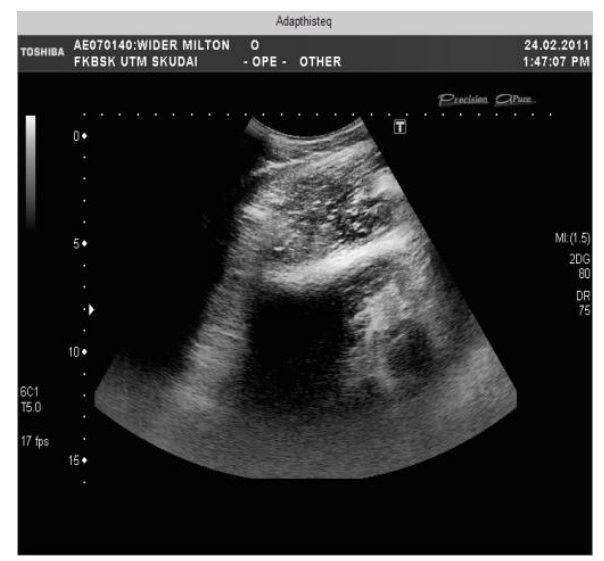

Fig-8: Result of Adaptive Histogram Equalization

According to these results, the adaptive histogram equalization method is used for image enhancement purpose.

Fig (9) and Fig (10) are the result of the segmentation step using the different thresholding methods.

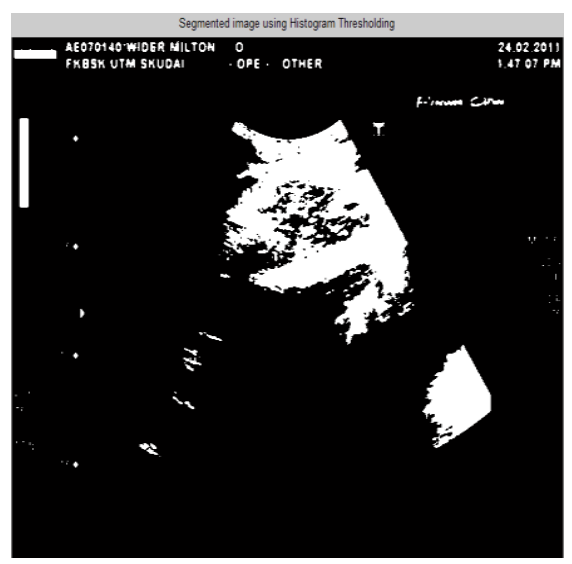

Figure-9: The result of the segmented image using Histogram Thresholding

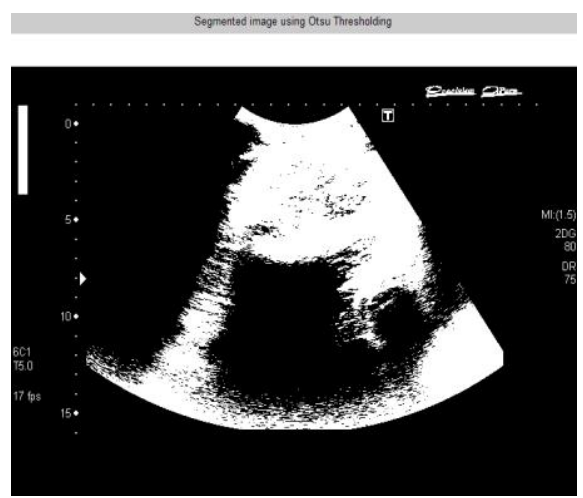

Fig-10: The result of the segmented image using Otsu's Thresholding

According to the result of images for the segmentation step, the histogram thresholding is the most appropriate method.

Fig (11) and Fig(12) are compared with the different edge detection method of canny edge detector and sobel edge detector.

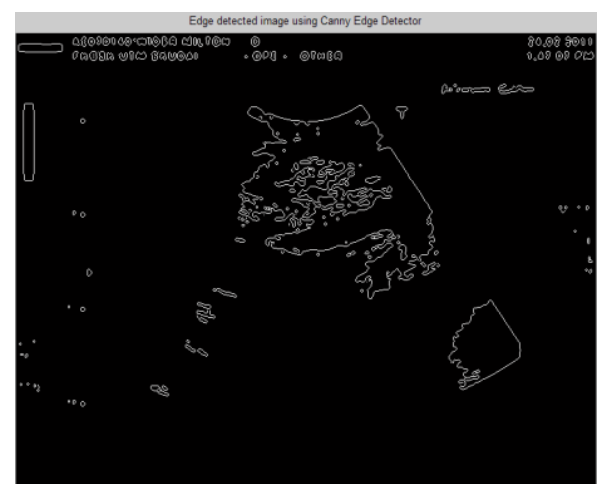

Fig-11: Edge detected image using Canny edge detector

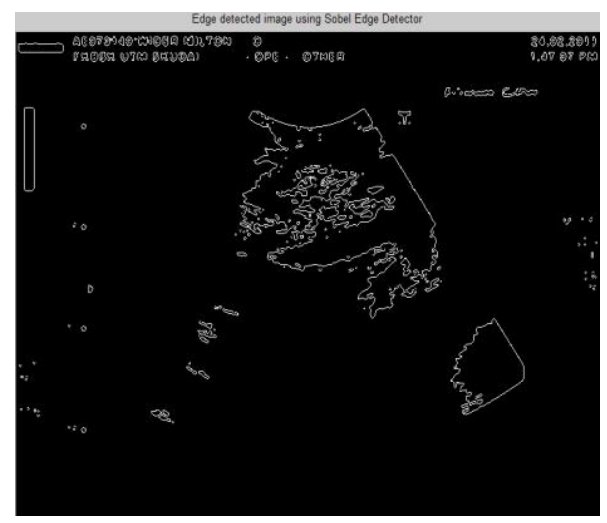

Fig-12: Edge detected image using Sobel edge detector

By the comparison of the edge detection, canny edge detector is more suitable than sobel edge detector for defining of ROI. 


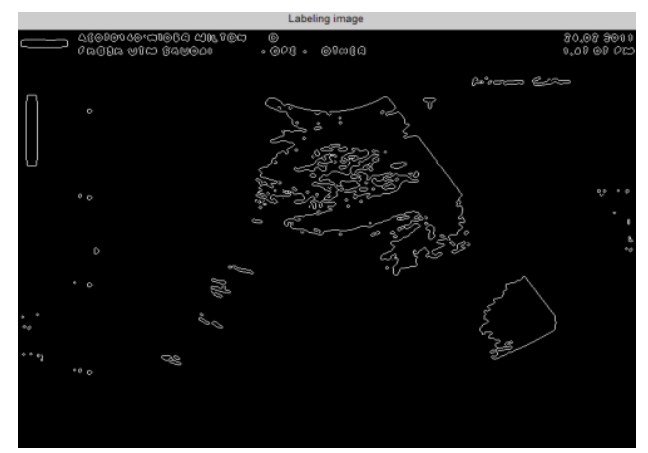

Fig-13: Appendix Labeling image

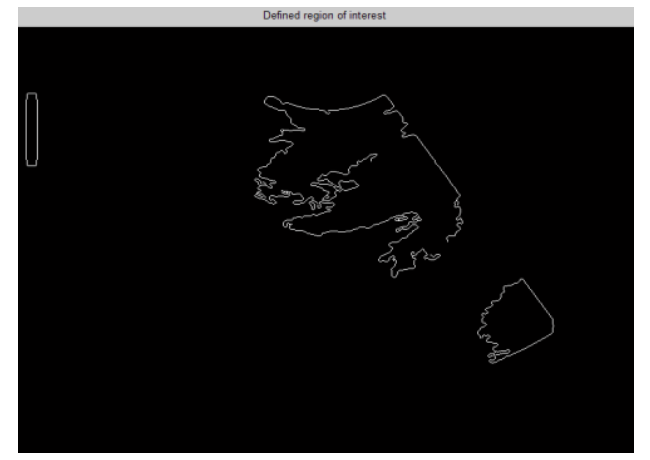

Fig-14: The result of ROI by using Canny Edge detector

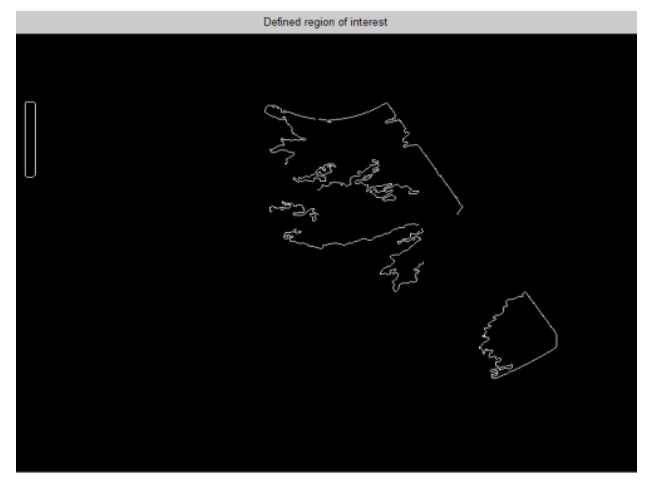

Fig-15: The result of ROI by using Sobel Edge detector

The original image is obtained from the Ultrasound as shown in figure 3 and figure 4 through 15 describes the step by step image processing.

From these figure, by the comparison of thresholding methods, histogram thresholding method is the most appropriate to segment out the appendix image. The canny edge detector is applied for this process. It can be said that the implemented process can detect clearly the outline of the appendix. This can make the evaluation easier by calculating the distance of the appendix. However the whole appendix cannot be detected. Some region of the appendix had been cut off due to the discontinuity of the pixel in the image after edge detection. This may be due to the poor image quality that captured by the ultrasound and inappropriate use of probes when examining which in turn give a low quality and blurry image.

\section{CONCLUSIONS}

Ultrasound appendix image processing using image segmentation was developed. Median filter is better noise reduction method in image enhancement state, can remove noise sharply and can provide an image like as original image. Canny edge detector is more suitable than Sobel edge detector. Sobel edge detector is cut some region of appendix therefore it cannot be used for determining the diameter of appendicitis. The outer diameter of appendicitis assumes $6 \mathrm{~mm}$ or more as a sign of acute appendicitis provides high sensitivity and specificity. The existence of appendicitis is seen at the end of the process using the distance measure on the ultrasound image.

\section{ACKNOWLEDGEMENTS}

The author would like to thank to her supervisor, Dr.Aung Soe Khaing for his valuable suggestion, and sharing his experience to write this research. The author is also thankful to the head of Department of Electronic Engineering, Mandalay Technological University for his encouragement, guidance and spending his precious time on her thesis work. And then, the author would like to express special thanks to the members and all of her teacher from Department of Electronic Engineering, Mandalay Technological University. Last, the author appreciates the help from Mandalay General Hospital for supporting a large collection of ultrasound appendix images which have been valuable for this research.

\section{REFERENCES}

[1] Appendicitis, Current Problem in Surgery, Volume42,Issue10, Octover2005

[2] M.Nadeesh, "Effective Identification of Acute Appendicitis Using Histogram Thresholding and Quasi-Euclidean Distance" International Journal of Computer and Organization Trends, Volume 31, Issue 3, 2013.

[3] Implementation of Ultrasound Appendix Image Detection, Online Available, www.en.wikipedia.org/wiki/Euclidean distance/accessed on 2013

[4] Signs and Symptoms of Appendicitis, Online Available, www.abdopain.com/symptoms of appendicitis.html/accessed on 29 May2013

[5] R. Balu, "Identification of Acute appendicitis Using Euclidean Distance on Sonographic Image",International Journal of Innovative Technology and Creative Engineering,Vol.1 No.7, JULY 2011

[6] P. John Konicki, and Erik B.Kulstad, "Diagnosis of appendicitis with ultrasound: case example", The Journal of Emergency Medicine,Vol.27,No.2, 2004 
[7] P. John Konicki, Erik B. Kulstad, DIAGNOSIS OF APPENDICITIS WITH ULTRASOUND: CASE EXAMPLE, The Journal of Emergency Medicine, Vol. 27, No. 2, pp. 187-189, 2004

[8] E. Supriyanto, M. Wider, and Y. M. Myint, "Ultrasound appendix image segmentation using histogram thresholding and image enhancement using noise filtering technique," in Proc. the $15^{\text {th }}$ WSEAS International Conference on Computers, World Scientific and Engineering Academy and Society (WSEAS): Corfu Island, Greece, 2011, pp. 223-227.

[9] Chai, H.Y., L.K. Wee, and E. Supriyanto, Ultrasound Images Edge Detection using Anisotropic Diffusion in Canny Edge Detector Framework. WSEAS TRANSACTIONS on BIOLOGY and BIOMEDICINE, April 2011. 8(2): p. 51-60

\section{BIOGRAPHIES}

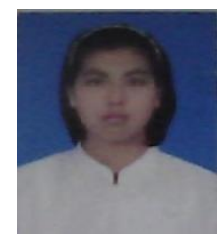

Kinsana Htwe received her Bachelor of Technology (B.Tech) degree in 2010 and Bachelor of Engineering (B.E) degree in 2011 in Electronics Engineering from Technological University, Meiktila, Myanmar. She is now Master of Engineering (M.E) thesis student in Mandalay Technological University, Myanmar. Her research interests image processing and biomedical.

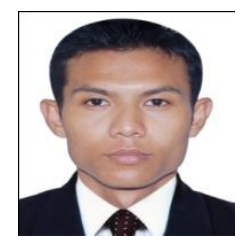

Aung Soe Khaing was born in Pyawbwe Township, Mandalay Division, Myanmar on 27.3.1982.He received Bachelor of Engineering in Electronics from Mandalay Technological University, Mandalay, Myanmar, in 2004 and Master of Engineering in Electronics from Yangon Technological University, Yangon, Myanmar, in 2006. He has continued his $\mathrm{PhD}$ dissertation in 2006. From October 2008 to September 2010, he was doing research on Spatial Frequency Analysis of the Human Brain at the Institute of Biomedical Engineering and Informatics, Technical University Ilmenau, Germany. He received his $\mathrm{PhD}$ in Electronic Engineering from Mandalay Technological University, Mandalay, Myanmar, in 2011.

He is now Associate Professor at Department of Electronic Engineering, Mandalay Technological University(MTU), Myanmar. His research interests include computer based Electrocardiogram (ECG) system, biomedical signal and image processing, bioinstrumentation and telemedicine.

Dr. Aung Soe Khaing was responsible for the ECG laboratory for the biomedical engineering students at the Institute of Biomedical Engineering and Informatics, Technical University Ilmenau, Germany from October 2008 to September 2010. 